\title{
A STRUCTURE THEOREM FOR SI-MODULES
}

\author{
by DINH VAN HUYNH and ROBERT WISBAUER
}

(Received 21 August, 1990)

An associative ring $R$ is called a left $S I$-ring if every singular left $R$-module is injective. In Goodearl [4] it is shown that these rings have a finite ring decomposition into a ring $K$ with $K /$ Soc $K$ left semisimple, and simple rings which are Morita equivalent to left SI-domains.

For an $R$-module $M$ denote by $\sigma[M]$ the full subcategory of $R$-Mod subgenerated by $M$. Extending the definition of SI-rings, we call an $R$-module $M$ an $S I$-module if every singular module in $\sigma[M]$ is $M$-injective. This also generalizes a similar notion in Yousif [11]. We obtain that every finitely generated, self-projective SI-module $M$ has a decomposition

$$
M=K \oplus V_{1} \oplus \cdots \oplus V_{n},
$$

with fully invariant submodules $K, V_{i}$, such that $K / \operatorname{Soc} K$ is a semisimple $R$-module, and, for $i=1, \ldots, n, \operatorname{End}_{R}\left(V_{i}\right)$ is a simple ring, and the category $\sigma\left[V_{i}\right]$ is equivalent to $T_{i}$-Mod for an SI-domain $T_{i}$.

1. Preliminary results. Let $R$ be an associative ring with unit and $R$-Mod the category of unital left $R$-modules. For $M \in R$-Mod we denote by $\sigma[M]$ the full subcategory of $R$-Mod whose objects are submodules of $M$-generated modules. $M$ is called self-projective if it is $M$-projective. Soc $M(\operatorname{resp} . \operatorname{Rad} M)$ denotes the socle (resp. the radical) of the module $M$. An $R$-submodule of $M$ is said to be fully invariant (or characteristic) if it is invariant under any $R$-endomorphism of $M$.

Morphisms are written on the opposite side to the scalars. For basic notions see [10].

The following elementary observations will be useful.

1.1. Proposition. Consider a self-projective $R$-module $M$ with $S=\operatorname{End}_{R}(M)$.

(1) If $\operatorname{Rad} M=0$, then $S$ has zero (Jacobson) radical.

(2) Assume $M$ is finitely generated. Then $M$ has no non-trivial fully invariant submodules if and only if $S$ is a simple ring.

Proof. (1) This follows from the fact that, for any simple homomorphic image $E$ of $M, \operatorname{Hom}_{R}(M, E)$ is a simple left $\operatorname{End}_{R}(M)$-module.

(2) For every ideal $I \subset S, M I \subset M$ is fully invariant. Since $M$ is self-projective, $I=\operatorname{Hom}_{R}(M, M I)$ by $[\mathbf{1 0}, 18.4]$ and hence $M I \neq M$ for $I \neq S$.

For every fully invariant submodule $U \subset M, \operatorname{Hom}_{R}(M, U)$ is an ideal in $S$.

If $K \subset M$ is an essential submodule, we write $K \unlhd M$.

Let $M$ and $N$ be $R$-modules. $N$ is called singular in $\sigma[M]$ or $M$-singular if $N \simeq L / K$ for some $L \in \sigma[M]$ and $K \unlhd L$ (see [9]).

By definition, every $M$-singular module belongs to $\sigma[M]$. For $M=R$ the notion $R$-singular is identical to the usual definition of singular for $R$-modules.

The class of all $M$-singular modules is closed under submodules, homomorphic images and direct sums (e.g. $[\mathbf{1 0}, 17.3$ and 17.4]). Hence every module $N \in \sigma[M]$ contains a largest $M$-singular submodule which we denote by $Z_{M}(N)$. The following properties of $M$-singular modules are shown in $[9,1.1]$ and $[8,2.4]$. 
1.2. Proposition. Let $M$ be an R-module.

(1) A simple $R$-module $E$ is $M$-singular or $M$-projective.

(2) If Soc $M=0$, then every simple module in $\sigma[M]$ is $M$-singular.

(3) If $M$ is self-projective and $Z_{M}(M)=0$, then the $M$-singular modules form $a$ hereditary torsion class in $\sigma[M]$.

We extend the definition of a left SI-ring (see [4]) to modules.

Definition. An $R$-module $M$ is called an $S I$-module if every $M$-singular module is $M$-injective.

In Yousif [11], $M$ is called an SI-module if every singular module in $R$-Mod is $M$-injective. Since $M$-singular modules are singular in $R$-Mod, this is a stronger condition than the one given above.

Though for $M=R$ the two notions coincide, in general SI-modules in our sense need not be SI-modules in the sense of Yousif (compare the example after $[9,2.2]$ ).

Let $T$ be a left SI-ring which is not left semisimple (for examples see [4], [1]), and $R$ the ring of lower triangular $(2,2)$-matrices over $T$. The map

$$
R=\left(\begin{array}{cc}
T & 0 \\
T & T
\end{array}\right) \rightarrow T, \quad\left(\begin{array}{ll}
a & 0 \\
b & c
\end{array}\right) \mapsto a,
$$

is a surjective ring homomorphism whose kernel is essential as left ideal in $R$. Hence every left $T$-module is singular as an $R$-module and all $T$-singular modules are $T$-injective, i.e. $T$ is a SI-module over $R$. Since $T$ is not left semisimple, not every $R$-singular module is $T$-injective. Hence $T$ is not an SI-module over $R$ in the sense of Yousif.

Every left module over a left SI-ring is an SI-module in the sense of Yousif and hence is an example of an SI-module in our sense.

In Smith [7], $R$ is called a left $R I C$-ring if every cyclic singular left $R$-module is injective. It is observed in [5, Corollary 5] that RIC-rings are SI-rings. By [9, 3.8 and 3.10] and [2, Lemma 2], we have more general statements in our next proposition which also include Proposition 3.1 and 3.6 in [4]. For this we recall two definitions.

An $R$-module $M$ is called hereditary in $\sigma[M]$ if every submodule of $M$ is projective in $\sigma[M]$ (see $[10,39.1]), M$ is a GCO-module (generalized co-semisimple) if every $M$-singular simple $R$-module is $M$-injective (see $[9,2.2])$.

1.3. Proposition. For a finitely generated, self-projective $R$-module $M$ the following conditions are equivalent:

(a) $M$ is an SI-module;

(b) every cyclic $M$-singular module is $M$-injective;

(c) $M / K$ is semisimple for every $K \unlhd M$ and $Z_{M}(M)=0$;

(d) $M$ is hereditary in $\sigma[M]$ and $M$-singular modules are semisimple;

(e) $M$ is a GCO-module, $M / \operatorname{Soc} M$ is noetherian and $\operatorname{Soc}(M / K) \neq 0$ for every $K \unlhd M$.

We will need the following lemma.

1.4. LemMA. Let $M$ be a self-projective SI-module with finite uniform dimension and $\operatorname{Rad} M=0$. Then $M$ contains no proper fully invariant submodule which is essential as an $R$-submodule. 
Proof. Assume $V \subset M$ is a fully invariant submodule and $V \unlhd_{R} M$. Since $\operatorname{Rad} M=0$, $S:=\operatorname{End}_{R}(M)$ has zero radical by Proposition 1.1. $\operatorname{Rad} M=0$ also implies that $\operatorname{Hom}_{R}(M, U) \neq 0$ for non-zero $U \subset M$ (see [8], p. 1475, (iv)). With this knowledge we derive from Theorem 3.7 in [8] that there exists a monomorphism $f: M \rightarrow V$, and since $M$ has finite uniform dimension, the image of every monomorphism in $S$ is essential in $M$. Hence the image of $f^{2}$ is essential in $M$. Therefore $M / M f^{2}$ is a semisimple module and the following exact sequence splits:

$$
0 \rightarrow M f / M f^{2} \rightarrow M / M f^{2} \rightarrow M / M f \rightarrow 0 .
$$

Applying the functor $\operatorname{Hom}_{R}(M,-)$ and the isomorphisms $S g \simeq \operatorname{Hom}(M, M g)$ for any $g \in S$ (see [10], 18.4), we obtain that $S f / S f^{2}$ is a direct summand in the $S$-module $S / S f^{2}$. Hence there exists a submodule $S f^{2} \subset U \subset S$ with $S f+U=S$ and $S f \cap U=S f^{2}$. This yields id $=r f+u$ for some $r \in S$ and $u \in U$ and hence $f=f r f+f u$. Since $f u \in S f \cap U=S f^{2}$ we finally have $f=f r f+s f^{2}$ for some $s \in S$. Since $f$ is monic this means $\mathrm{id}=f r+s f$ and $M=M f r+M s f \subset V$.

2. Structure theorem. Let us first describe uniform SI-modules with zero socle.

2.1. Proposition. For a finitely generated, self-projective $R$-module $M$, the following are equivalent:

(a) $M$ is a uniform SI-module with Soc $M=0$;

(b) $M$ is a self-generator and $\operatorname{End}_{R}(M)$ is a left SI-domain which is not a division ring.

Under this condition, $M$ has no fully invariant submodules and $\operatorname{End}_{R}(M)$ is a simple ring.

Proof. (a) $\Rightarrow$ (b). If $M$ is an SI-module with zero socle, all simple modules in $\sigma[M]$ are $M$-singular (by Proposition 1.2), hence $M$-injective and $M$-generated. Therefore $M$ is a projective generator in $\sigma[M]$. This implies that $\sigma[M]$ is equivalent to $S$-Mod (see [10, 18.5 and 46.2]) and $S$ is a left SI-ring.

Since $Z_{M}(M)=0$, every $f \in \operatorname{End}_{R}(M)$ is a monomorphism.

(b) $\Rightarrow$ (a). The functor $\operatorname{Hom}_{R}(M,-)$ is an equivalence.

The last part follows from Lemma 1.4 and Proposition 1.1.

Now we investigate the decomposition of SI-modules with zero socles.

2.2. THEOREM. For a finitely generated, self-projective $R$-module $M$ and $S=$ End $_{R}(M)$, the following are equivalent:

(a) $M$ is an SI-module and $\operatorname{Soc} M=0$;

(b) $M$ is a generator in $\sigma[M]$ and $S$ is a left $S I$-ring with zero left socle;

(c) $M=M_{1} \oplus \ldots \oplus M_{n}$, with $M_{i}$ minimal fully invariant submodules, and $\sigma\left[M_{i}\right]=$ $\sigma\left[L_{i}\right]$ for some finitely generated, self-projective and uniform SI-module $L_{i}$ with zero socle;

(d) $M=M_{1} \oplus \ldots \oplus M_{n}$, with $M_{i}$ fully invariant submodules, $\operatorname{End}_{R}\left(M_{i}\right)$ simple rings and $\sigma\left[M_{i}\right]$ equivalent to $T_{i}$-Mod, for left SI-domains $T_{i}$ which are not division rings.

Proof. (a) $\Leftrightarrow$ (b). As observed in the proof of Proposition 2.1, (a) implies that $M$ is a projective generator in $\sigma[M]$. Hence $\sigma[M]$ is equivalent to $S$-Mod (see $[10,18.5$ and 46.2]) and $M$ is an SI-module if and only if $S$ is a left SI-ring. 
(a) $\Rightarrow$ (c). As noted above, $M$ is a generator in $\sigma[M]$, and by Proposition $1.3, M$ is noetherian and hereditary in $\sigma[M]$. Every essential submodule of $M$ is an intersection of maximal submodules, and $\operatorname{Soc} M=0$ implies $\operatorname{Rad} M=0$ and $S$ has zero radical by Proposition 1.1.

By Theorem 3.7 in [8], the endomorphism ring of the $M$-injective hull $\hat{M}$ is semisimple artinian, i.e. $\operatorname{End}_{R}(\hat{M})=T_{1} \oplus \ldots \oplus T_{n}$ with simple artinian rings $T_{i}$. Denoting by $e_{i}$ the unit in $T_{i}$, we have $e_{1}+\ldots+e_{n}=\operatorname{id}_{\hat{M}}$ and, since the $e_{i}$ are in the center of $\operatorname{End}_{R}(\hat{M})$,

$$
\hat{M}=\hat{M} e_{1} \oplus \ldots \oplus \hat{M} e_{n}
$$

is a decomposition into fully invariant submodules. The intersection $M_{i}:=M \cap \hat{M} e_{i}$ is a fully invariant submodule of $M$ and $M_{1} \oplus \ldots \oplus M_{n} \unlhd_{R} M$. As we have seen in Lemma 1.4 , this means

$$
M_{1} \oplus \ldots \oplus M_{n}={ }_{R} M \text {. }
$$

Since $\operatorname{Hom}_{R}\left(M_{i}, M_{j}\right)=0$ for $i \neq j$, we observe that $\hat{M} e_{i}$ is the injective hull of $M_{i}$ in $\sigma\left[M_{i}\right]$. Moreover $M_{i}$ is a self-projective self-generator with $Z_{M}(M)=0$ and, again applying Theorem 3.7 in [8], we know that $\operatorname{End}_{R}\left(\hat{M} e_{i}\right) \simeq T_{i}$ is the classical left quotient ring of $\operatorname{End}_{R}\left(M_{i}\right)$. Hence $\operatorname{End}_{R}\left(M_{i}\right)$ has no non-trivial central idempotents and $M_{i}$ has no non-trivial decomposition into fully invariant submodules.

To study properties of the summands $M_{i}$ we may assume that $M$ itself has no non-trivial decomposition into fully invariant submodules. We want to show that $M$ has no proper fully invariant submodules.

Let $X \subset M$ be fully invariant. First consider a non-zero $R$-submodule $Y \subset M$ with $X \cap Y=0$. We show that $X$ and $Y$ do not have isomorphic uniform submodules: assume, for a uniform submodule $U \subset X$, there exists a monomorphism $g: U \rightarrow Y$. Since $\operatorname{Hom}_{R}(M, U)$ is a non-zero left ideal in $S$ and $\operatorname{Rad} S=0$, we can find $f: M \rightarrow U$ with $f^{2} \neq 0$ and hence $(U) f \neq 0$. Then $(U) f g \subset Y$ and, by the invariance of $X$, also $(U) f g \subset X$ implying $(U) f=0$, a contradiction.

Now let $\left\{Y_{\lambda}\right\}_{\Lambda}$ denote the family of all submodules of $M$, with no uniform submodules isomorphic to submodules of $X$, and put $Y=\sum_{\Lambda} Y_{\lambda}$.

Assume $Y$ contains a uniform submodule $U$ isomorphic to a submodule of $X$. Since $M$ is hereditary in $\sigma[M]$, we may suppose $U \subset \oplus Y_{\lambda}$, and we conclude that $U$ has an isomorphic copy in one of the $Y_{\lambda}^{\prime}$ 's (compare $[10,39.7]$ ), a contradiction. Obviously, $X \cap Y=0$ and, by the above observation, $X \oplus Y \unlhd_{R} M$.

Hereditariness of $M$ also implies that, for any $f \in S,(Y) f$ has no uniform submodules isomorphic to submodules in $X$. Hence $(Y) f \subset Y$, i.e. $Y$ and $X \oplus Y$ are fully invariant in $M$. By Lemma 1.4, we have $X \oplus Y=M$. This means by assumption $X=M$.

Now choose a uniform submodule $U \subset M$ and a non-zero $f \in \operatorname{Hom}_{R}(M, U)$. Then $L:=(M) f$ is uniform and $M$-projective. The trace $\operatorname{Tr}(L, M)$ of $L$ in $M$ is fully invariant and hence $\operatorname{Tr}(L, M)=M$, implying $\sigma[M]=\sigma[L]$.

(c) $\Rightarrow$ (d). Each of the $L_{i}$ is a progenerator in $\sigma\left[M_{i}\right]=\sigma\left[L_{i}\right]$ (see proof of $(\mathrm{a}) \Leftrightarrow(\mathrm{b})$ ). Hence $\sigma\left[M_{i}\right]$ is equivalent to $T_{i}$-Mod where $T_{i}:=\operatorname{End}_{R}\left(L_{i}\right)$ is a left SI-domain by Proposition 2.1.

According to Proposition 1.1, $\operatorname{End}_{R}\left(M_{i}\right)$ is a simple ring. 
(d) $\Rightarrow$ (b). By the given equivalences, every $M_{i}$ is an SI-module and $\sigma\left[M_{i}\right]$ contains an $M_{i}$-projective generator $L_{i}$ with zero socle. Then also $M_{i}$ has zero socle and is a progenerator in $\sigma\left[M_{i}\right]$ (see proof of $(\mathrm{a}) \Leftrightarrow(\mathrm{b})$ ), and $\operatorname{End}_{R}\left(M_{i}\right)$ is a left SI-ring. As a product of these rings, End ${ }_{R}(M)$ is also a left SI-ring.

REMARK. For the proof of (b) $\Rightarrow$ (c) we could have used part of Goodearl's structure theorem for left SI-rings in $[4,3.11]$. For $M=R$ our proof provides an alternative to Goodearl's proof of the corresponding part.

Finally we are ready to prove the following extension of Goodearl's characterization of Sl-rings in [4, 3.11].

2.3. Structure Theorem. For a finitely generated, self-projective $R$-module $M$ and $S=\operatorname{End}_{R}(M)$, the following are equivalent:

(a) $M$ is an SI-module;

(b) $Z_{M}(M)=0$ and $M$ has a decomposition

$$
M=K \oplus V_{1} \oplus \ldots \oplus V_{n}
$$

with fully invariant submodules $K, V_{i}$, such that $K / \operatorname{Soc} K$ is a semisimple $R$-module, and, for $i=1, \ldots, \operatorname{End}_{R}\left(V_{i}\right)$ is a simple ring and the category $\sigma\left[V_{i}\right]$ is equivalent to $T_{i}$-Mod, for an SI-domain $T_{i}$ which is not a division ring.

Under the given conditions, $S$ is a left SI-ring.

Proof. (a) $\Rightarrow(\mathrm{b})$. Assume $M$ is an SI-module. As already observed in Proposition 1.3, $M$ is hereditary and $\bar{M}:=M / \operatorname{Soc} M$ is noetherian.

As noted in Proposition 1.2, the $M$-singular modules form a torsion class in $\sigma[M]$. Let $K$ denote the $R$-submodule $\operatorname{Soc} M \subset K \subset M$ such that $K / \operatorname{Soc} M$ is the torsion submodule of $\bar{M}$ in this torsion theory. Since $\operatorname{Soc} M$ is fully invariant in $M$ and $K / \operatorname{Soc} M$ is fully invariant in $M / \operatorname{Soc} M, K$ is fully invariant in $M$.

By construction, Soc $K=\operatorname{Soc} M$. Also $K / \operatorname{Soc} M$ is an SI-module and Soc $M \unlhd K$ since $K$ is projective in $\sigma[M]$ ( $M$ hereditary). Hence $K /$ Soc $M$ is semisimple by 1.3 and $M$-injective by assumption. Therefore

$$
\bar{M}=K / \operatorname{Soc} M \oplus N / \operatorname{Soc} M
$$

for some $R$-submodule $N \subset M$ containing $\operatorname{Soc} M$. Since $\operatorname{Soc} M$ is a fully invariant submodule, $\bar{M}$ is self-projective. As $M / L$ is semisimple for $L \unlhd M$, and Soc $M$ is the intersection of all $L \unlhd M$, we conclude $\operatorname{Rad} \bar{M}=0$.

Hence $M / K=N /$ Soc $M$ is a self-projective SI-module with zero radical. By definition of $K, M / K$ contains no $M$-singular submodules. Therefore every simple submodule of $M / K$ is $M$-projective by Proposition 1.2. Since $\operatorname{Soc} M \subset K$, we conclude $\operatorname{Soc}(M / K)=0$.

Denote by $\left\{H_{\lambda}\right\}_{\wedge}$ the family of all submodules of $M$ with $\operatorname{Soc} H_{\lambda}=0$ and set $V=\sum_{\Lambda} H_{\lambda}$. Since all simple submodules of $V \subset M$ are $M$-projective (by Proposition 1.2) and $\oplus_{\Lambda} H_{\lambda}$ has zero socle, also Soc $V=0$ and $K \cap V=0$. The $M$-projectivity of simple submodules of $M$ also implies that, for every $f \in S$, (V)f has zero socle and hence $(V) f \subset V$, i.e. $V$ is fully invariant. It is obvious from the definitions and the properties derived that $\operatorname{Soc} M \oplus V \unlhd K \oplus V \unlhd_{R} M$ and that $K \oplus V$ is a fully invariant submodule of M. 
Passing to the factor module, we have that $(K \oplus V) / K$ is a fully invariant submodule of $M / K$ which is essential as an $R$-submodule. Recalling the properties of $M / K$ shown above, by Lemma 1.4, this implies $K \oplus V=M$.

The composition of $V$ is now obtained from Theorem 2.2.

(b) $\Rightarrow$ (a). Obviously, for every essential submodule $U \subset M, M / U$ is semisimple and hence $M$ is an SI-module by Proposition 1.3.

It remains to show that $S$ is a left SI-ring. Since $M$ is hereditary in $\sigma[M], S$ is left semi-hereditary by $[\mathbf{1 0}, 39.14]$ and hence left non-singular.

By $(b), \operatorname{End}_{R}(M)=\operatorname{End}_{R}(K) \times \operatorname{End}_{R}\left(V_{1}\right) \times \cdots \times \operatorname{End}_{R}\left(V_{n}\right)$. In the proof of Theorem 2.2 we have shown that all $\operatorname{End}_{R}\left(V_{i}\right)$ are left SI-rings. Therefore it is enough to show that $S_{1}=\operatorname{End}_{R}(K)$ is also a left SI-rng.

From the exact sequence $0 \rightarrow \operatorname{Soc} M \rightarrow M \rightarrow \bar{M} \rightarrow 0$, we derive the exact sequence

$$
0 \rightarrow \operatorname{Hom}(M, \operatorname{Soc} M) \rightarrow S \rightarrow \operatorname{Hom}(M, \bar{M}) \rightarrow 0 .
$$

Since $\bar{M} \approx K / \operatorname{Soc} K$ is semisimple, $\operatorname{Hom}(M, \bar{M})$ is a semisimple left $S$-module. From $\operatorname{Hom}(M, \operatorname{Soc} M) \subset \operatorname{Soc} S$ we conclude that $S / \operatorname{Soc} S$ is left semisimple and $S$ is a left S1-ring by Proposition 1.3.

Remark. For $M=R$, our Structure Theorem yields Goodearl's Structure Theorem for SI-rings (see [4, 3.11]), which was also proved in Theorem 2.7 of Baccella [1] in a different way.

Obviously any SI-module is a GCO-module (compare 1.3). By our Structure Theorem we obtain that self-projective GCO-modules with descending chain condition on essential submodules are SI-modules. Referring to $[9,3.11]$ we have the following corollary.

2.4. Corollary. For a finitely generated, self-projective $R$-module $M$, the following are equivalent:

(a) $M$ is an SI-module with dcc on essential submodules;

(b) $M$ is a GCO-module with dcc on essential submodules;

(c) $M /$ Soc $M$ is semisimple and $Z_{M}(M)=0$.

Acknowledgement. The first author is grateful to the Alexander von Humboldt Foundation for financial support during his stay at the Mathematical Institute of the University of Düsseldorf while this paper was written.

\section{REFERENCES}

1. G. Baccella, Generalized $V-$ Rings and Von Neumann regular rings, Rend. Sem. Mat. Univ. Padova 72 (1984) 117-133.

2. Nguyen V. Dung, Dinh van Huynh and R. Wisbauer, Quasi-injective modules with acc or $d c c$ on essential submodules, Arch. Math. (Basel) 53 (1989) 252-255.

3. Dinh van Huynh, P. F. Smith and R. Wisbauer, A note on $G V$-modules with Krull dimension, Glasgow Math. J. 32 (1990), 389-390. (1972)

4. K. R. Goodearl, Singular torsion and the splitting properties, Mem. Amer. Math. Soc. 124

5. B. Osofsky and P. F. Smith, Cyclic modules whose quotients have all complement modules direct summands, J. Algebra 139 (1991), 342-354. 
6. S. S. Page and M. F. Yousif, Relative injectivity and chain conditions, Comm. Algebra 17 (1989) 899-924.

7. P. F. Smith, Rings characterized by their cyclic modules, Canad. J. Math. 31 (1979) 93-11.

8. R. Wisbauer, Localization of modules and the central closure of rings, Comm. Algebra 9 (1981), 1455-1493.

9. R. Wisbauer, Generalized co-semisimple modules, Comm. Algebra. 18 (1990), 4235-4253.

10. R. Wisbauer, Foundations of module and ring theory (Gordon and Breach, New York, 1991).

11. M. F. Yousif, SI-modules, Math. J. Okayama Univ. 28 (1966) 133-146.

Institute of Mathematics

P.O. BOX 631

HANOI, VIETNAM

INSTITUTE OF MATHEMATICS

UNIVERSITÄTSSTR. 1

4000 Düsseldorf, Germany 